East African Medical Journal Vol. 86 No. 7 July 2009

HYPO-PHOSPHATAEMIA IN CHILDREN UNDER FIVE YEARS WITH KWASHIORKOR AND MARASMIC KWASHIORKOR

D. Kimutai, MBChB, MMed, Consultant Paediatrician, Ministry of Health, P. O. Box 30016, Nairobi, Kenya, E. MalecheObimbo, MBChB, MMed, MPH(Epi), Paediatrician, Epidemiologist and Senior Lecturer, Department of Paediatrics and Child Health, College of Health Sciences, University of Nairobi, P.O. Box 19676-00202, Nairobi, Kenya, R. Kamenwa, MBChB, MMed, FNIC, Paediatrician, Neonatologist and Lecturer, Kenyatta National Hospital, P.O. Box 20723-00202 Nairobi, Kenya and F. Murila, MBChB, MMed, FNIC, Paediatrician, Neonatologist and Lecturer, Department of Paediatrics and Child Health, College of Health Sciences, University of Nairobi, P.O. Box 19676-00202, Nairobi, Kenya

Request for reprints to: Dr. D. Kimutai, Department of Paediatrics and Child Health, College of Health Sciences, University of Nairobi, P.O. Box 19676-00202, Nairobi, Kenya

\title{
HYPO-PHOSPHATAEMIA IN CHILDREN UNDER FIVE YEARS WITH KWASHIORKOR AND MARASMIC KWASHIORKOR
}

\author{
D. KIMUTAI, E MALECHE-OBIMBO, R. KAMENWA and F. MURILA
}

\begin{abstract}
Background: Severe malnutrition contributes up to $50 \%$ of childhood mortality in developing countries is frequently characterised by electrolyte depletion, including low total body phosphate. During therapeutic re-feeding, electrolyte shift from extracellular to intra-cellular compartments may induce hypo-phosphataemia (hypo-P) with resultant increased morbidity and mortality. This biochemical imbalance is under-recognised, and the frequency of this problem among African malnourished children is unclear.

Objectives: To determine the magnitude of hypo-phosphataemia in children under five years of age presenting to Kenyatta National Hospital with kwashiorkor and marasmic kwashiorkor and to evaluate the relationship between hypo-phosphataemia and nutritional intervention during the first five days of treatment.

Design: Short longitudinal survey.

Setting: The General Paediatric wards of the Kenyatta National Hospital (KNH), Nairobi.

Subjects: Children under five years of age presenting with kwashiorkor or marasmic kwashiorkor at $\mathrm{KNH}$ were recruited into the study.

Main outcome measures: Low serum phosphate level $(<1.20 \mathrm{mmol} / 1)$ and patient outcome (survival or death) during the first five days of treatment.

Results: One hundred and sixty five children were enrolled between June 2005 and February 2006 of which $107(64 \%)$ had kwashiorkor and $58(36 \%)$ had marasmic kwashiorkor. They were of mean age 20 months (range 3-60), and $95(58 \%)$ were male. The prevalence of hypo-phosphataemia was $86 \%$ on admission, increased to $90 \%$ and $93 \%$ on day one and two respectively, and then declined to $90 \%$ by the fourth day. At admission $6 \%$ were hypo-phosphataemic, increasing to $18 \%$ and $22 \%$ on day one and two respectively, and declining to $11 \%$ by day four. On admission mean serum phosphate was below normal at $0.91 \mathrm{mmol} / 1$, declined significantly to $0.67 \mathrm{mmol} / 1$ and to a nadir of $0.63 \mathrm{mmol} / 1$ after the first and second day of treatment respectively, then rose slightly to $0.75 \mathrm{mmol} / 1$ on the fourth day ( $\mathrm{p}<0.001$ comparing each follow-up mean level with the admission level). There was a positive association between severity of nadir serum phosphate level and mortality $(p=0.028)$. There were no deaths among children with normal nadir serum phosphate levels. However, among children with mild, moderate and severe nadir hypo-phosphataemia, 8,14 and $21 \%$ died respectively. Children with dermatosis and hypomagnesaemia showed a trend for association with mortality ( $\mathrm{p}=0.082$ and 0.099 respectively).

Conclusion: Hypo-phosphataemia is frequent among children with kwashiorkor and marasmic kwashiorkor presenting at KNH. Serum phosphate levels decline significantly during the first two days of nutritional intervention, and severity of hypo-phosphataemia is directly associated with mortality.
\end{abstract}




\section{INTRODUCTION}

Nutritional deficiencies constitute a major public health problem in the tropical and subtropical regions of the world. Among them, protein energy malnutrition (PEM) is the most widespread condition. In Africa, the estimated prevalence of severe PEM ranges between 1.7-9.8\% (1). According to the Kenya Demographic Health Survey (KDHS) of 2003, 20\% of children below five years of age were underweight and $4 \%$ were severely underweight (2).

Despite improved medical care, mortality of children with severe PEM remains high at 10-20\% (3). Mortality in kwashiorkor is reported by Fechner et al (4) to range between 10 and $50 \%$. This high mortality is mainly found in sub-Saharan Africa despite implementation of standard treatment protocols (5). Observations from the Paediatric Emergency Ward at $\mathrm{KNH}$ showed that malnutrition accounted for $7 \%$ of admissions and $15 \%$ of mortality (6).

A number of biochemical changes in the blood and tissues have been described in PEM (1). The most common electrolyte abnormalities are hypo-phosphataemia (hypo-P), hypokalaemia and hypomagnesaemia, and they have been associated with mortality in the initial days of treatment $(7,8)$. Phosphorus is an essential macro-mineral generally found in the human body as part of the phosphate anion, $80 \%$ of which is intra-cellular and principally found in bones. The phosphate, ion is involved in the energy pathways of the cell. Although it is available in most foods, it tends to be depleted in certain disease states like malnutrition.

Hypo-phosphataemia has become a topic of concern due to increasing evidence that it may be associated with serious morbidity (9). Hypo-Phas been found in patients being therapeutically re-fed after severe weight loss (9). Re-feeding syndrome is defined as the occurrence of severe fluid and electrolytes shifts and their associated complications in malnourished patients undergoing enteral or parenteral re-feeding $(10,11)$. The main abnormalities observed include hypo-P, hypokalaemia and hypomagnesaemia $(10,11)$. Re-feeding syndrome is one of the few conditions associated with profound hypo-P due to intra-cellular shifts of phosphorus (9).Itis known that malnourished children are depleted of phosphorus due to renal loss and inadequate intake during starvation. During refeeding, there is a sudden introduction of carbohydrate whichstimulates insulin release. Insulin subsequently increases cellular uptake of glucose, phosphate and water into cells and this results in a severe hypo-P, a central component of the re-feeding syndrome.

Re-feeding syndrome is associated with high mortality. Patients at risk include those with anorexia nervosa, chronic alcoholism, chronic malnutrition, the elderly and oncology patients. Friemen et al (12) found that 10\% (6 of 60) of children presenting with kwashiorkor in South Africa had severe hypo-P and that the metabolic disturbance was associated with dehydration and death. Severe hypo-P (serum phosphate $<0.33 \mathrm{mmol} / \mathrm{l})$, occurred in eight $(12 \%)$ of 68 children with kwashiorkor within 48 hours of admission and five $(63 \%)$ of the eight children died compared with $13(22 \%)$ of the 60 without severe hypo-P $(\mathrm{p}<0.02)$. Therefore it was concluded that severe hypo-P seems to be common and life threatening in children with kwashiorkor (13). In the same study, dermatosis and dehydration were significantly correlated with severe hypo-P but these clinical signs could not reliably predict fatal outcomes.

A systematic review of literature revealed 27 children that developed re-feeding syndrome after oral or enteral feeding, nine (33\%) of whom died as a direct result of complications of this syndrome (10). Cautious progressive nutrition rehabilitation and careful monitoring of vital signs, electrolyte levels and fluid balance are essential for prevention of morbidity and mortality from this syndrome (14).

It was with this background that we determined to evaluate the magnitude of hypo-P among children under five years of age admitted to $\mathrm{KNH}$ with kwashiorkor or marasmic kwashiorkor, and to evaluate association between hypo-P and outcome during the first five days of treatment.

\section{MATERIALS AND METHODS}

This was a short longitudinal survey between June 2005 and February 2006 at KNH, a teaching and referral hospital in Nairobi, Kenya. All children under five years of age admitted to the hospital with kwashiorkor or marasmic kwashiorkor during the study period, whose mothers / guardians consented, were eligible for inclusion. Patients were excluded if they had rickets, diabetes mellitus or if they were on antacid, diuretic or insulin therapy.

Outcomes of interest included prevalence and severity of hypo-P, and survival versus death during five day follow up. Approval to carry out the study was obtained from the KNH Ethics and Research Committee.

Clinical and laboratory procedures: The investigator visited the study area daily from 8.00 am to 5.00 pm and consecutively enrolled subjects meeting the inclusion criteria and consenting to participate until the desired sample size was achieved. The initial evaluation included a complete history and physical examination. Demographic and clinical data of each subject were obtained and recorded.

Subjects were classified according to the Welcome Trust classification as kwashiorkor if they had oedema and weight for age fell between 60 to $80 \%$ of expected; they were classified as marasmic kwashiorkor if they had oedema and their weight for age was less than $60 \%$ of expected. 
During this initial contact, 2 millilitres of blood was collected, placed in a labelled plain plastic container and promptly transported to the Renal Unit laboratory within the hospital for analysis. Blood sampling was done serially at admission (day 0 ), and on days one, two and four after admission.

Patients follow-up: In the wards, patients were managed for malnutrition by the ward clinicians according to hospital guidelines. This entailed the use of special milk which is a mixture of cow's milk, sugar, corn oil and eggs that contains approximately $100 \mathrm{Kcal} / \mathrm{dl}$ when ingested. The starting volumes usually were $100 \mathrm{mls} / \mathrm{kg} /$ day divided into eight equal three hourly feeds. Infections and other comorbidities were managed routinely following the usual hospital guidelines. The patients were reviewed every morning for up to seven days and outcome recorded as either 'alive' or 'died' at one week post-admission. For those who died, the time from admission to death was recorded.

Laboratory investigations: Serum was separated within two hours to avoid haemolysis and thereafter biochemical analysis performed using the automated method. Baseline serum phosphorus, albumin, magnesium and calcium were determined. Serum phosphorus was subsequently assayed on days one, two and four post-admission.

Data management: Data were managed using the Statistical Package for Social Sciences Software (SPSS version 11). The prevalence of hypo-P was determined and categorised into normal phosphate (1.20$2.30 \mathrm{mmol} / 1)$, or mild $(0.80-1.19 \mathrm{mmol} / 1)$, moderate $(0.3-30.79 \mathrm{mmol} / \mathrm{l})$ and severehypo- $\mathrm{P}(<0.33 \mathrm{mmol} / 1)$.
Thelowestever serum phosphate level reached (nadir phosphate) was computed for all the children and the day on which the nadir occurred noted. The mean serum phosphatelevel was calculated among children who died and in those that survived on each day and compared using independent $\mathrm{t}$-test.

Continuous variables were compared using students t-test, and categorical values were compared using Chi-square test, Fisher's Exact test and Chisquare for linear trends as appropriate. Level of significance was set at $<0.05$.

\section{RESULTS}

Description of the cohort: Out of 165 children enrolled into the study between June 2005 and February 2006, 107 (64\%) had kwashiorkor and 58 (36\%) had marasmic kwashiorkor. Males comprised 58\% (95 of $165)$ with a male to female ratio of 1.4: 1 . The mean age of the children in the study was 20 months (range 3-60) with mean ages of 23 months for children with kwashiorkor and 19 months for those with marasmic kwashiorkor. Common morbidities included dehydration in $104(63 \%)$, diarrhoea in $93(56 \%)$ and dermatosis in $62(38 \%)$ of children (Table 1$)$.

Prevalence and severity of hypo-phosphataemia: On the day of admission 142 of 165 children had hypo-P, giving a prevalence of $86 \%$ and of these nine $(6 \%)$ had severe hypo-P. Prevalence of hypo-P was similarly high among children with kwashiorkor and those with marasmic kwashiorkor (89 versus $99 \%$ respectively, $\mathrm{p}=0.166)$. Among the 142 hypo-phosphataemic children, $90(54 \%)$ had mild, $43(26 \%)$ had moderate and nine $(6 \%)$ had severe hypo-P (Table 2).

Table 1

Demographic and clinical characteristics of children with kwashiorkor at the Kenyatta National Hospital ( $n=165)$

\begin{tabular}{llcl}
\hline Characteristic & & No. & $(\%)$ \\
\hline Sex & Males & 95 & 58 \\
Age (months) & Mean (Range) & 20 & $3-60$ \\
Class of malnutrition & Kwashiorkor & 107 & 64 \\
& Marasmic- kwashiorkor & 58 & 36 \\
Hydration status & Dehydration & 104 & 63 \\
Frequency and duration of & Diarrhoea & 94 & 57 \\
diarrhoea & Acute & 60 & 36 \\
& Chronic & 34 & 21 \\
Presence of infection & Yes & 134 & 81 \\
Presence of dermatosis & Present & 62 & 38 \\
IV Glucose given & Yes & 46 & 28 \\
\hline
\end{tabular}


Table 2

Prevalence of hypo-phosphataemia at presentation in children with kwashiorkor presenting at Kenyatta National Hospital $(n=165)$

\begin{tabular}{|c|c|c|c|c|c|c|}
\hline \multirow[t]{2}{*}{$\begin{array}{l}\text { Presence or absence of low } \\
\text { serum phosphate }\end{array}$} & \multicolumn{2}{|c|}{ Kwashiorkor } & \multicolumn{2}{|c|}{$\begin{array}{l}\text { Marasmic } \\
\text { kwashiorkor }\end{array}$} & \multicolumn{2}{|c|}{ All patients } \\
\hline & No. & $(\%)$ & No. & $(\%)$ & No. & $(\%)$ \\
\hline \multicolumn{7}{|l|}{ Hypo-phosphatemia present } \\
\hline Mild & 57 & 53 & 33 & 57 & 90 & 54 \\
\hline Moderate & 25 & 23 & 18 & 31 & 43 & 26 \\
\hline Severe & 7 & 7 & 2 & 3 & 9 & 6 \\
\hline Total hypo-phosphataemia & 89 & 83 & 53 & 91 & 142 & 86 \\
\hline Hypo-phosphataemia absent & 18 & 17 & 5 & 9 & 23 & 14 \\
\hline Total & 107 & 100 & 58 & 100 & 165 & 100 \\
\hline
\end{tabular}

Table 3

Prevalence of hypo-phosphataemia during nutritional intervention in children with kwashiorkor at Kenyatta National Hospital

\begin{tabular}{|c|c|c|c|c|c|c|c|c|}
\hline $\begin{array}{l}\text { Presence or absence of low } \\
\text { serum phosphate }\end{array}$ & $\begin{array}{l}\text { Day } \\
\text { No. }\end{array}$ & $\begin{array}{l}0 \\
(\%)\end{array}$ & $\begin{array}{l}\text { Day } \\
\text { No. }\end{array}$ & $\begin{array}{l}1 \\
(\%)\end{array}$ & $\begin{array}{l}\text { Day } \\
\text { No. }\end{array}$ & $\begin{array}{l}2 \\
(\%)\end{array}$ & $\begin{array}{l}\text { Day } \\
\text { No. }\end{array}$ & $\begin{array}{l}4 \\
(\%)\end{array}$ \\
\hline \multicolumn{9}{|l|}{ Hypo-phosphataemia present } \\
\hline Mild & 90 & 54 & 45 & 28 & 44 & 28 & 52 & 34 \\
\hline Moderate & 43 & 26 & 71 & 44 & 68 & 43 & 68 & 45 \\
\hline Severe & 9 & 6 & 29 & 18 & 35 & 22 & 17 & 11 \\
\hline Total hypo-phosphataemia & 142 & 86 & 145 & 90 & 147 & 93 & 137 & 90 \\
\hline Hypo-phosphataemia absent & 23 & 14 & 16 & 10 & 11 & 7 & 15 & 10 \\
\hline Total & 165 & 100 & 161 & 100 & 158 & 100 & 152 & 100 \\
\hline
\end{tabular}

During nutritionalintervention, theoverall prevalence of hypo-P increased to $90 \%$ on day one, to $93 \%$ on day two, and dropped to $90 \%$ on day four. The prevalence of severe hypo-P tripled to $18 \%$ on day one, further increased to $22 \%$ on day two, and declined to $11 \%$ on day four (Table 3).

We report on mean phosphorous levels from admission day to the fourth post-admission day. The mean serum phosphate level dropped significantly from baseline level of $0.91 \mathrm{mmol} / 1$ to $0.67 \mathrm{mmol} / \mathrm{L}$ and $0.63 \mathrm{mmol} / 1$ after one and two days of treatment respectively $(p<0.001)$. Phosphate levels thereafter rose to $0.75 \mathrm{mmol} / 1$ on day four (Figure 1 ).
The majority of children $(47 \%)$ experienced their lowest serum phosphate level (nadir) on day two, whilst $21 \%, 22 \%$ and $10 \%$ experienced nadir levels on days zero, one and four respectively.

Outcome following nutritional intervention: Twenty one $(13 \%)$ children died during the first five days in hospital. During follow-up, we noted that the mean serum phosphate level on any given day was lower among those who died than among survivors although this difference did not achieve statistical significance (Figure 2). 
Figure 1

Trends of mean serum phosphate level during intervention in children with kwashiorkor at Kenyatta National Hospital

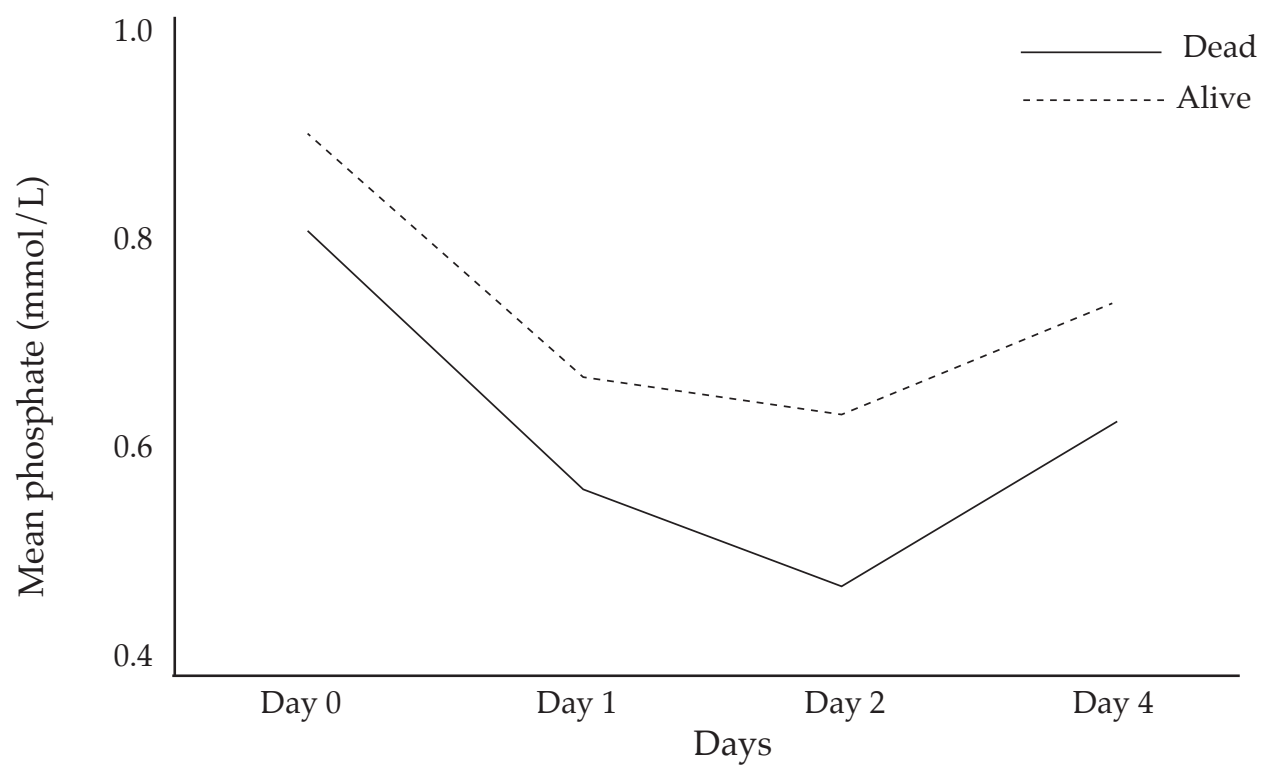

The nadir serum phosphate level was established for each child and used to categorise children into either normal nadir serum phosphate or mild, moderate or severe hypophosphataemic nadir. Mortality rose significantly with increasing severity of nadir hypo-P from no deaths among children with normal serum nadir phosphate, to $8 \%, 14 \%$ and $21 \%$ mortality among children with mild, moderate and severe nadir hypo-P respectively $(\mathrm{p}=0.028)$ (Figure 3 ).

In univariate analysis there was a trend for severe hypo-P among children with versus without dermatosis (67\% vs $36 \%$ respectively, $p=0.082$ ) and among children with versus without low magnesium levels (78\% vs $48 \%$ respectively, $\mathrm{p}=0.099$ ) at admission. There was no association however between malnutrition class, sex, age, hydration status, diarrhoea, infection, intravenous glucose administration, albumin, calcium and severe hypo- $\mathrm{P}$ on admission.

\section{DISCUSSION}

Hypo-P occurs frequently among children less than five years of age with kwashiorkor and marasmic kwashiorkor admitted to the paediatric wards of $\mathrm{KNH}$. An overall prevalence of hypo-P of $86 \%$ at presentation and of severehypo-P of $6 \%$ was observed. This is consistent with what has been found in other studies especially in Africa. Frieman et al (12) in
South Africa found $10 \%$ of all children presenting with kwashiorkor to have severe hypo-P and that this metabolic disturbance was associated with dehydration and death. The overall prevalence of hypo-P in their study was, however, lower at $57 \%$.

A fairly recent Malawian study found an overall prevalence of hypo-P at presentation to be high at $76 \%$ with a prevalence of severe hypo-P of $12 \%$ which was positively associated with death (13).

Similar high prevalence of $50 \%$ of hypo-P was observed in two studies done in the paediatric intensive care units (16). Commonly associated with hypo-P in these patients were malnutrition, re-feeding syndrome, sepsis, trauma, and diuretic and steroid therapies. The patients in the current study were all severely malnourished and being therapeutically re-fed and so the finding of a high prevalence of hypo-P both at presentation and during follow up is not surprising.

We speculate that the prevalence of severe hypo-P of $6 \%$ might not portray the true picture of the magnitude of severe phosphorus deficiency in this cohort. This is because during starvation, in order to maintain normal phosphate levels there is catabolic release of phosphate from intra-cellular stores which is subsequently lost in the urine and therefore significant total body phosphate depletion can occur despitenormal serum phosphate levels (15). Severely malnourished children most often have had 
a long-term relatively low phosphate intake which predisposes them to significant total body phosphate depletion $(9,16)$.

Up to $86 \%$ of our patients already had hypo-P on admission and hence it is not surprising to see a progression to severe hypo-P with nutritional repletion. Afzal et al (10) reported 27 children ( $<18$ years) that developed re- feeding syndrome characterised by a fall in phosphate after enteral feeding with high calorie feeds. In general, these children were malnourished (weight range 45-75\% of ideal weight for height) with seven of them having kwashiorkor. The cohort in our study comprised severely malnourished children with a weight for age below $80 \%$ who were similarly undergoing nutritional intervention with a high calorie diet and therefore, the development of re-feeding hypo-P is not surprising.

This study demonstrated a relatively higher mean serum phosphate of $0.91 \mathrm{mmol} / 1$ on admission with only $6 \%$ of the patients found to have severe hypo-P. On follow up, phosphate levels significantly dropped with a simultaneous rise in percentage of severe hypo-P, peaking after two days. Manary et al (13) observed a similar trend where $12 \%$ of patients developed severe hypo-P within 48 hours of nutritional intervention. From our cohort, $22 \%$ of the patients attained severe levels as early as 48 hours and a rise in mean phosphate level was observed on day four.

Marik et al (17) studied a heterogeneous group of 62 intensive care unit adult patients who were undernourished and had undergone a period of starvation lasting more than 48 hours. They found $21(34 \%)$ of their patients to have developed re-feeding hypo-P with a nadir serum phosphate attained after about two days. These hypo-phosphataemic patients had a longer period of mechanical ventilation and hospital stay. In their study, the finding of a nadir serum phosphate after two days resembles the findings in our cohort and also demonstrates that refeeding hypo-P can accompany fairly short periods of starvation due to intra-cellular phosphate shifts.

Similar trends have been observed in adult studies. Weinsier et al (18) reported two adult cases with severe malnutrition that developed severe hypo$P$ within 48 hours of total parenteral nutrition. Their serum phosphate levels similarly showed an upward trend on day four. Both patients died soon afterwards from complications of the re-feeding syndrome. It is believed that re-feeding hypo-P significantly contributed to their deaths due to the close temporal relationship between declining serum phosphate level and their acute cardiac decompensation. In our study, $21 \%$ (8 of 38) of patients with severe hypo-P died. Mortality rates ranging from 63 to $100 \%$ have been found in patients who developed the re-feeding syndrome $(13,18)$.

From this cohort, it was demonstrated that there is an increasing mortality with increasing severity of nadir serum phosphate level. A similar finding has been observed in other studies elsewhere $(12,13,18)$. However, the mortality in this cohort may be slightly lower and we speculate that this may be attributable to the erratic and inadequate supply of feeds administered to the children in this setting. This scenario of sub-optimal intake of calories tends to be inadvertently protective against the effects of the re-feeding syndrome. Therefore, in most instances, refeeding syndrome is treated by cautious progressive nutrition achieved by a reduction in volume and caloric density of the feeds $(10,14,19,20)$.

In the current study, it was demonstrated that a modest proportion of $19 \%$ of the deaths occurred a day after the mean nadir serum phosphate. The deaths on admission could be attributable to the initial complications of malnutrition observed on admission such as hypoglycaemia, hypothermia, and dehydration $(7,21)$. The rest of the mortalities are distributed throughout the week. There are no similarly designed studies to compare our findings with. However, Manary et al (13) attributed five of eight $(63 \%)$ of mortalities to hypo-P in the first 48 hours of admission. They only followed up their cohort to 48 hours after admission. Other reports which comprise mainly case studies, have reported mortality to occur even later than the first week $(10,18)$.

There were a number of limitations to our study. Although we explored the demographic, clinical and laboratory correlates of hypo-P, the study was not designed to answer that question and therefore was under-powered to demonstrate clearly the presence or absence of associations. Due to logistical reasons, the amount of feed prescribed could not be measured during the study period. Confounders that may have contributed to mortality include hypoglycaemia, hypothermia, dehydration and infections. Follow-up of patients to discharge was not done as only the first week was considered. Therefore, full outcome during the admission for every child was not measured.

We concluded that the prevalence of hypo-P at admission was high at $86 \%$, with $54 \%, 26 \%$ and $6 \%$ having mild, moderate and severe levels respectively. We also concluded that in our study population an increase in the prevalence of hypo-P after nutritional intervention, rose from $86 \%$ at baseline to $90 \%$ and 93\% at day one and two respectively, and was still high at $89 \%$ on day 4 . Severe hypo-P prevalence similarly rose to $18 \%$ and $22 \%$ on day one and two respectively and dropped to $10 \%$ on day four. Our 
findings demonstrates a positive association between severity of nadir hypo-P and mortality, with an observed mortality of $0 \%$ among children with normal nadir phosphate and 8,14 and $21 \%$ among children with mild, moderate and severe nadir hypo-P. The findings of this study will also inform the formulation of future feeding guidelines.

With such a high prevalence of severe hypo-P, and with there being no reliable clinical method for identification of this metabolic disturbance, we recommend that serum phosphate should at least be measured at admission and on day two (13). Our findings suggest that there may be value in providing routine phosphate supplementation while re-feeding severely malnourished children. There is need to further evaluate theefficacy and safety of phosphorus supplementation in kwashiorkor and marasmic kwashiorkor children. Future studies should also evaluate the effect of the gradual introduction and progression in calories on the reduction of hypo-P.

\section{ACKNOWLEDGEMENTS}

To Mr. C. Chepkok who processed all the samples at the KNH Renal Laboratory; Mr. M. Mwangi of Kenya Medical Research Institute for his assistance in data analysis; to all the doctors, nurses and nutritionists of $\mathrm{KNH}$ paediatrics wards who assisted in enrollment of study subjects, to the parents and children who accepted to participate in this study.

\section{REFERENCES}

1. Reddy, V.Protein-Energy Malnutrition. In: Diseases of children in the subtropics and tropics. Eds, Stanfield, P., Brueton, M., Chan, M., et al. Edward Arnold, London. 1991. 4th edition, pp 335-338.

2. Ministry of Health (Kenya), Kenya Demographic and Health Survey Data. Central Bureau of Statistics (CBS): Nairobi, 2003.

3. Seear, M., Hendricks, M. and Bamford, L. The child who is failing to grow. In: A Manual of Tropical Pediatrics, Ed. Seear, M. Cambridge University Press, Cambridge. 2000; 3: 25-30.

4. Fechner, A., Catharina, C., Bohme, et al. Antioxidant status and Nitric oxide in the malnutrition syndrome kwashiorkor. Peadiatr. Res. 2001; 49: 237-243.

5. Brewster, D.R., Manary, M. J. and Graham, S. M. Case management of kwashiorkor: an intervention project at seven nutrition rehabilitation centres in Malawi. Eur. J. Clin. Nutr. 1997; 51:139-147.
6. Mortality data. Paediatric Emergency Ward: KNH, 1987.

7. Beers, M.H. and Berkow, R.eds: Nutritional Disorders: Malnutrition. In: The Merck Manual. 17th ed, Whitehouse Station. N.J. Merck \& Co. 1999; 28-32.

8. Andrew, L. Protein energy malnutrition: A review. eMedicine J. [serial online]. 2003.

9. Knochel, J. P. The pathophysiology and clinical characteristics of severe hypo-phosphatemia. Arch. Intern. Med. 1977; 137: 203-220.

10. Afzal, N, A., Addai, S., Fagbemi, A., et al. Refeeding syndrome with enteral nutrition in children: a case report, literature review and clinical guidelines. Clin. Nutr. 2002; 21: 515-520.

11. Solomon, S.M. and Kirby, D. S. The refeeding syndrome. A review. J. Parenter. Enteral. Nutr. 1990; 14: 90-97.

12. Friemen, I., Pettifor, I. M. and Moodley, G.M. Serum phosphorus in protein energy malnutrition. J. Pediatr. Gastroenterol. Nutr. 1982; 1: 547-550.

13. Manary, M., Hart, C. A. and Whyte, M. Severe hypo-Phosphatemia in children with kwashiorkor is associated with increased mortality. J. Paediatr. 1998; 133:789-791.

14. Monti, M. and Lamy, O. Hypo-Phosphatemia and refeeding syndrome: a severe and undiagnosed adverse effect. Schweiz Rundsch Med. Prax. 2003; 92: 1744-1747.

15. Berkelhammer, C. and Bear, R. A. A clinical approach to common electrolyte problems. 3. HypoPhosphatemia. Can. Med. Assoc. 1984; 130: 17-23.

16. Fernando, S. M., Heitor, P. L., Juliana, F. et al. HypoPhosphatemia in critically ill children. Rev. Hosp. Clin. 2004; 59.

17. Marik, P. E, and Bedigian, M.K. Refeeding hypoPhosphatemia in critically ill patients in an intensive care unit. A prospective study. Arch. Surg. 1996; 131: 1043-1047.

18. Weinsier, R. L. and Krumdieck, C. L. Death resulting from overzealous total perenteral nutrition: the refeeding syndrome revisited. Am. J. Clin. Nutr. 1981; 34: 393-399.

19. Kohn, Michael, Golden, et al. Cardiac arrest and delirium: Presentations of the refeeding syndrome in severely malnourished adolescents with anorexia Nervosa . J. Adol. Health. 1998; 22: 239-243.

20. Fisher, M., Simpser, E. and Schneider, M. HypoPhosphatemia secondary to oral refeeding in anorexia nervosa. Int. J. Eat. Disord. 2000; 28: 181-187.

21. World Health Organisation. Management of severe malnutrition: a manual for physicians and other senior health workers, WHO: Geneva, 1999. 